\title{
Effect of Contrast, Stimulus Density, and Viewing Distance on Multifocal Steady-State Visual Evoked Potentials (MSVs)
}

\author{
Siti N. Abdullab, ${ }^{1-3}$ Nada Aldablawi, ${ }^{1,4}$ Yanti Rosli, ${ }^{5}$ Vaegan, ${ }^{1,6}$ Mei Ying Boon, ${ }^{1}$ and \\ Ted Maddess ${ }^{3}$
}

Purpose. We investigated the effects of image contrast, stimulus density, and viewing distance upon a multifocal steady-state visual evoked potential (MSV) method.

Methods. Fourteen adults with normal vision (mean age $=27.0$ \pm 6.6 years; 6 males) participated in the study. Each of the stimulus regions of the multifocal ensembles presented a contrast modulated grating, displaying spatial and temporal frequencies that evoke the spatial frequency doubling illusion. All subjects were tested at five contrasts: 0.06, 0.11, 0.22, 0.45, and 0.89 ; viewed at 16,32 , and $128 \mathrm{~cm}$. A multivariate linear model estimated factors for each stimulus region, recording channel, number of stimuli (9 or 17 regions), and sex; and covariates for contrast, and octaves of viewing distance, and age.

REsults. The responses per unit area for the 17-region display were significantly larger than for the 9-region display $(P<$ $10^{-12}$ ). The contrast-response function could be described by a power law with exponent $0.068(P<0.008)$. The effect of viewing distance was small but significant: response amplitude dropped by $-0.17 \pm 0.03 \mathrm{~dB}$ per octave of viewing distance $(P$ $<10^{-6}$ ), or $10 \%$ over 8 octaves.

Conclusions. The response per unit area indicated that cortical folding diminishes responses to larger stimuli. Viewing distance did not greatly affect response amplitude. This suggested that we can use similar, but scaled, stimuli to study central and peripheral disease. The rapidly saturating contrast responses imply that there would be nothing lost from testing at contrasts as low as $20 \%$ given that higher, saturating contrasts might mask visual field defects. (Invest Ophthalmol Vis Sci. 2012;53:5527-5535) DOI:10.1167/iovs.11-9325

From the ${ }^{1}$ School of Optometry and Vision Science, University of New South Wales, Sydney, Australia; the ${ }^{2}$ Orthoptic Unit, Department of Ophthalmology, Raja Isteri Pengiran Anak Saleha (RIPAS) Hospital, Bandar Seri Begawan, Brunei; the ${ }^{3}$ Australian Research Council Centre of Excellence in Visual Science, John Curtin School of Medical Research, Australian National University, Canberra, Australia; the ${ }^{4}$ Optometry Department, King Saud University, Applied Medical Sciences, Riyadh, Saudi Arabia; and the ${ }^{5}$ School of Diagnostic and Applied Health Sciences, Faculty of Health Sciences, Universiti Kebangsaan Malaysia, Kuala Lumpur, Malaysia.

${ }^{6}$ Deceased November 10, 2008.

Supported by grants from the Australian Research Council (ARC) through the ARC Centre of Excellence in Vision Science (CE0561903) and a PhD scholarship from the Government of Brunei Darussalam (SNA).

Submitted for publication December 14, 2011; revised May 20 and June 28, 2012; accepted July 4, 2012.

Disclosure: S.N. Abdullah, None; N. Aldahlawi, None; Y. Rosli, None; Vaegan, None; M.Y. Boon, None; T. Maddess, Seeing Machines (F), P

Corresponding author: Ted Maddess, Australian Research Council Centre of Excellence in Vision Science (ACEVS), John Curtin School of Medical Research (Bldg 131), Australian National University, Canberra ACT 0200, Australia; ted.maddess@anu.edu.au.
The multifocal visual evoked potential (mfVEP) was first 1 introduced by Baseler and Sutter, ${ }^{1}$ and permits localized visual field (VF) deficits to be quantified. Here we examine multifocal steady-state visual evoked potentials (MSV). The basis for the method has been reported in two previous studies, ${ }^{2,3}$ and is derived from a multifocal electroretinogram (mfERG) method that has been shown to have predictive value in diagnosing glaucoma. ${ }^{4,5}$ One reason for using a steady-state method here is to permit multifocal stimuli that all display the spatial frequency doubling (FD) illusion, ${ }^{6}$ like the stimuli used in the suprathreshold test of the Frequency Doubling Technology (FDT) perimeter (Carl Zeiss, Meditec, Dublin, CA).

Visual evoked potential (VEP) responses are affected by cortical folding, particularly by the calcarine sulcus. The geometry of Brodmann area 17 (V1) and the sulcus mean that when stimulating two parts of the VF they may produce largely equal but opposite electric field vectors on either side of the sulcus, thus, producing no net recorded response at some points on the scalp..$^{7-10}$ Less severe interference between response generators located in smaller cortical folds can also reduce VEP amplitudes. To get around these cortical folding effects researchers have attempted to use 200 or more very small multifocal stimuli in an attempt to isolate responses from every part of the occipital cortical sheet, ${ }^{11}$ but this requires long recording durations. Here, we will examine the effect on response amplitudes of dicing the area of our stimulus regions by a factor of 2; thus, producing stimuli that sample the VF at two densities.

Another factor that influences VEP responses is cortical magnification, ${ }^{12-14}$ which is largely determined by the retinal ganglion cell (RGC) density of the retina, ${ }^{15}$ and the fact that each millimeter squared of $\mathrm{V} 1$ receives approximately the same number of afferent fibers; thus, the part of the V1 representing the fovea is larger than more peripheral areas. ${ }^{12,16,17}$ The mapping is approximately log-polar. ${ }^{16,18,19}$ Under that mapping, if a person is fixating on the center of an object that looms toward them, such that its VF area increases by $x$, the cortical representation of the object maintains its size and shape and translates along the log (VF radius) axis of the cortex by an amount proportional to $\log (x)$. Thus, one might expect little change in VEP response amplitude for radial scaling of peripherally presented stimuli that correspond to the effects of looming. Accordingly, we also examined the effect on the MSVs of the viewing distances: 16,32 , and $128 \mathrm{~cm}$.

Finally, we have previously reported $^{2}$ strongly saturating CRFs for the MSV method, and this could affect the results obtained here. Therefore, the three viewing distances and two stimulus densities were assessed at five contrast levels in each of 14 subjects.

\section{Materials ANd Methods}

\section{Subjects}

The study included 6 males and 8 females (mean \pm SD, $27.0 \pm 6.6$ years). The subjects had vision corrected to $6 / 6$ or better (Bailey-Lovie 

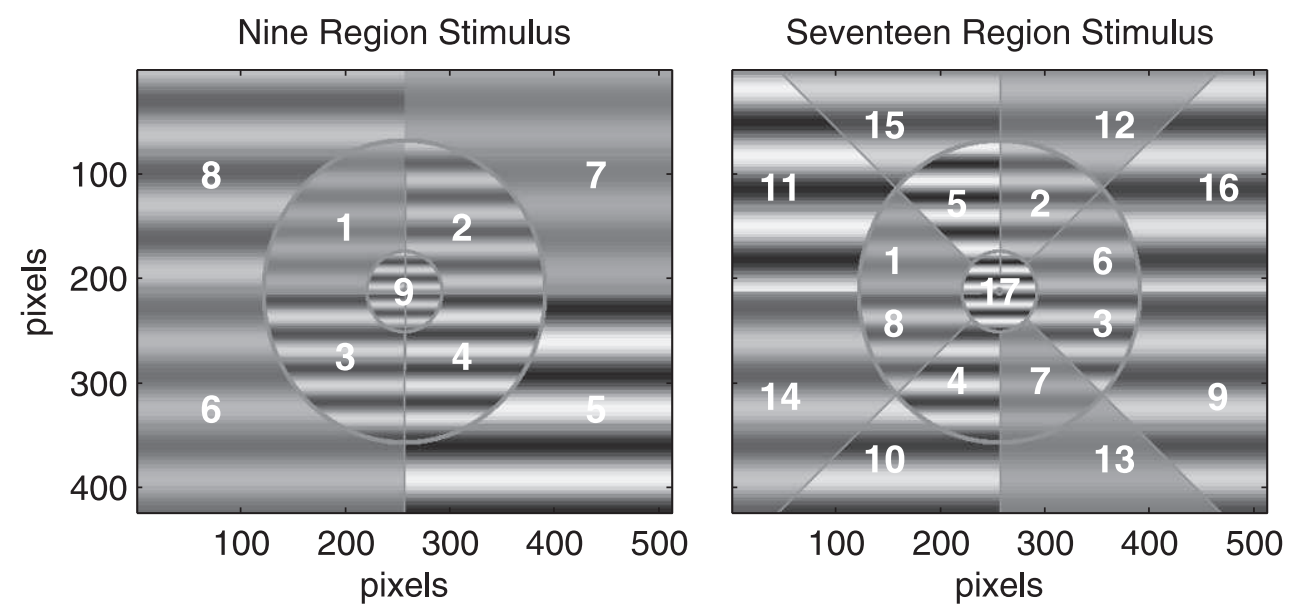

FIGURE 1. Illustration of the 9 (left) and 17 region (right) FDT-like stimuli used in the experiments. The contrasts of the gratings are as if the stimulus sequence had been stopped partway through the experiment. At the standard viewing distance of $16 \mathrm{~cm}$, the spatial frequency was fixed within each ring, having a central value of $0.25 \mathrm{cpd}, 0.125 \mathrm{cpd}$ for the middle ring, and $0.0625 \mathrm{cpd}$ for the outer ring. The numbers labeling each region indicate the increasing order of the temporal frequencies of the stimuli (see Fig. 2). A circular cardboard mask defined the outer border of the outer ring. The angles subtended at the retina reduced as viewing distance was altered from nearer $16 \mathrm{~cm}\left(84.4^{\circ}\right)$, and $32 \mathrm{~cm}\left(48.8^{\circ}\right)$ to the farthest $128 \mathrm{~cm}\left(12.9^{\circ}\right)$.

chart), and refractive errors less than \pm 6 dioptres of spherical and 1.5 diopters of cylindrical correction. All subjects certified that they were not medicated for any systemic or ocular disorders. Their undilated right eyes were tested, and their left eyes were occluded. All procedures were fully explained to the subjects and informed written consent was obtained. Subjects were treated in accordance with the Declaration of Helsinki and the experiments were regulated by the Human Research Ethics Committee of the University of New South Wales.

\section{The MSV Stimuli}

The MSV stimuli were generated by a graphics card (Vista; Truevision, Shadeland Station, IN) and were displayed on a HP1230 CRT monitor (Hewlett-Packard, Palo Alto, CA) with a resolution of 512 by 424 pixels. The mean luminance of the screen was $54.5 \mathrm{~cd} / \mathrm{m}^{2}$. The stimuli were sinusoidal achromatic gratings having bars oriented horizontally (Fig. 1). Grating contrasts were corrected for the room lighting $3.80 \mathrm{~cd} /$ $\mathrm{m}^{2}$ ). The two densities of stimulus ensembles displayed either 9 or 17 regions per VF (Fig. 1). There were 2-mm gaps between each of the stimulus regions, which were designed to remove short range interactions. ${ }^{20}$ A piece of white cardboard with a 290-mm diameter hole was mounted on the monitor to define an outer circular border resulting in three concentric rings of stimuli as illustrated in Figures $2 \mathrm{~A}$ and $2 \mathrm{~B}$. At the viewing distance of $16 \mathrm{~cm}$, each ring had a fixed spatial frequency (SF), central 0.25 cycles per degree (cpd), middle $0.125 \mathrm{cpd}$, and outermost at 0.0625 cpd (Fig. 1). The octave intervals of SF scaling with eccentricity accords with RGC densities. ${ }^{21}$ A red fixation spot, 2 pixels square, was presented at the center of the screen.

\section{Stimulus Frequencies and Data Acquisition}

The display rate of the monitor was 101.50 frames/s. The VEP signals were sampled at four times the video frame rate synchronous with the onset of each frame. The stimulus length was 2048 frames for the 9region stimulus, and 4096 frames for the 17-region stimulus. The durations of the two types of stimuli ensemble were, therefore, $20.18 \mathrm{~s}$ (i.e., 2048/101.50) and $40.35 \mathrm{~s}$, producing frequency resolutions ( $d f$ ) of 0.0496 or $0.0248 \mathrm{~Hz}$.

The incommensurate contrast modulation frequencies of the 9 and 17 regions occurred in the Fourier spectrum at the zero based indices jfreq $=444,448,453,459,466,476,490,502,510$, and 888, 892, 897, 903, 910, 920, 934, 946, 954, 973, 994, 1023, 1053, 1088, 1121, 1176,
1192, respectively. The actual temporal frequencies, therefore, were in the range 22.02 to $25.28 \mathrm{~Hz}$ for the 9-region stimuli (Fig. 2A), and 22.02 to $29.56 \mathrm{~Hz}$ for the 17 -region stimuli (Fig. 2B), and were, thus, within bands $3.27 \mathrm{~Hz}(25.28-22.01)$ and $7.53 \mathrm{~Hz}(29.54-22.01)$ wide for the 9- and 17-region stimuli, respectively. The resulting response frequencies were, therefore, in a band double the width of the stimulus frequencies. Within the response band for the 9-region stimuli there were 45 unique second order response frequencies and 88 noise (i.e., nonsignal $=$ noise) frequencies that were used to estimate the signal to noise ratios (SNR) within the narrow band of response frequencies, and significance of the responses was based on an F-statistic for each response as previously described. ${ }^{5}$ For the 17 -region stimuli there were 605 frequencies, 452 of which were noise frequencies. The 17-region design is similar to that used for a dichoptic MSV that had 16-stimulus regions in total. ${ }^{3}$ The gratings modulated at the temporal and spatial frequencies described here exhibit the FD illusion for VF locations like those used here. ${ }^{22}$ Importantly, the selected frequencies in the stimulus band mean that any frequencies generated by second order interactions between regions are separate from the second harmonic and noise frequencies. ${ }^{2,3}$

\section{Testing Conditions}

Stimuli were presented at three different viewing distances, 16, 32, and $128 \mathrm{~cm}$ (Figs. 2, 3). Measurements for each viewing distance and stimulus density were repeated at five contrast steps: $0.06,0.11,0.22$, 0.45 , and 0.89 . The three distances, five contrasts, and two densities of display ( 9 or 17 regions) made for 30 stimulus conditions per subject. Each subject viewed the 30 conditions in a random presentation order. Two repeats of each of the 30 conditions were taken and the complex valued response coefficients were averaged on each of the three recording channels, ensuring that signals with consistent phase were enhanced. A companion study showed that two repeats is adequate for estimating adequate effect sizes when measuring contrast-response functions (CRFs). ${ }^{2,3}$

In order to understand the effects of viewing distance on recorded VEPs it is necessary to understand that for the outer two rings of the stimulus the cortical representation in V1 is well approximated by a log-polar transformation (i.e., where the coordinates are the logarithm of the distance from fixation and the polar angle around fixation). A log-polar transform has advantages for the recognition of looming objects that are centered on the fovea. As mentioned previously, when objects loom (expand) by a factor $x$ in the retinal image, their cortical 

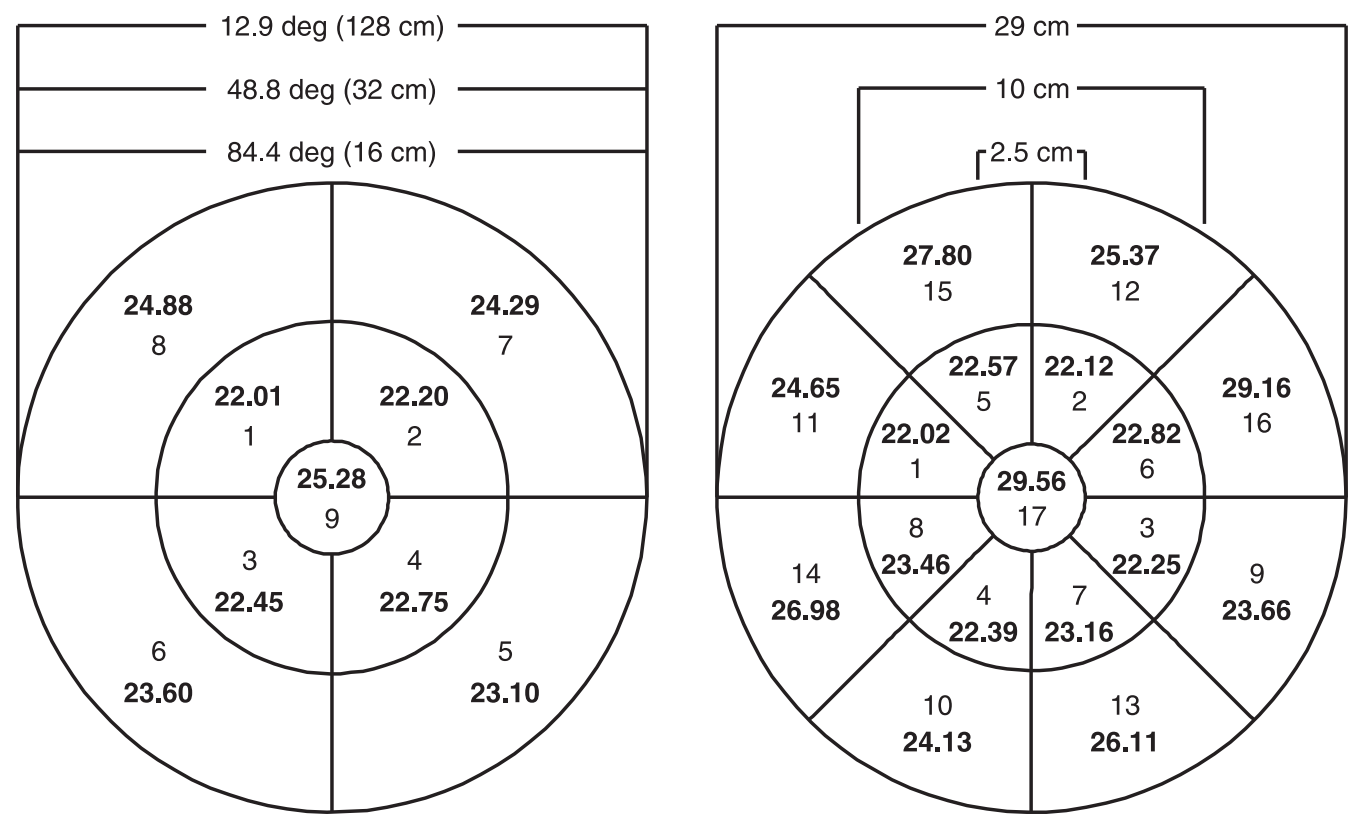

Figure 2. A schematic representation of the 9- (left) and 17-region (right) stimulus ensembles. The numbers in boxes are the region numbers, and those outside the boxes are the stimulus contrast modulation frequencies (hertz). The angles subtended, viewing distances, and physical size of each ring are also illustrated.

representation remains the same size and just translates along the logradius axis by an amount proportional to $\log (x)$. This representational invariance probably aids the visual system to recognize looming objects as they approach.

Figure 3 illustrates this effect for the outer two rings of the stimulus ensemble over the 8-fold change of viewing distance used here (Figs. 3A, 3C, 3E). The calculated log-polar representations on the cortical sheet of an area like $\mathrm{V} 1$ are shown in Figures 3B, 3D, and 3F. The transformation calculations were done with Matlab (The Mathworks, Natick, MA). Notice also that the cortical spatial frequency of the grating stimuli, in terms of cycles per millimeter of cortex, also remains fixed as the viewing distance changes. The number of afferent inputs per millimeter squared of the cortical sheet is fairly constant, so this scaling also means that each cycle of the stimulus is always spanned by an equal number of cortical afferent receptive fields. Thus, given that the cortical excitation by the stimuli remains is relatively unchanged for the different view distances, the expectation is that VEP amplitudes should be little affected by changes in viewing distance. If correct this could be a useful property for assessing different areas of the VF by stimuli of various scales.

\section{Electrode Positioning and Recording}

VEPs were amplified 100,000 times using a four channel amplifier (built at Australian National University by Mark Snowball) and were bandpass filtered between 3 and $100 \mathrm{~Hz}$. The amplified data were acquired using a Labmaster (Scientific Solutions Inc., Solon, $\mathrm{OH}$ ) board. A three channel recording configuration was employed (Fig. 4). The inion was set as reference for the first two channels, ${ }^{23}$ and electrodes $(\mathrm{C}$ and $\mathrm{D})$ were placed lateral to the inion. ${ }^{23,24}$ Electrode placement lateral to the inion has been reported to enhance the signals from the center of the field as well as those just below the horizontal VF meridian. ${ }^{23,24}$ Channel 3, C-D, was a differential record with D being the reference for $\mathrm{C}$, an arrangement that has been reported to be sensitive to movement ${ }^{25}$ (i.e., at the approximate position of visual area MT). The subjects in this study were grounded with yoked electrodes placed behind both ears at the mastoids.

Electrode impedance was kept to less than $5 \mathrm{~K} \Omega$. Eight millimeter gold cup electrodes were held firmly with electrode paste (Elfix; Nihon
Kohden, Tokyo, Japan) and taped in position with 1 inch Micropore Tape (3M Corporation, Maplewood, MN).

\section{Results}

The major effects of the number of regions, contrast, channels, and viewing distance were explored by plotting the means of the response amplitudes across various stimulus parameters. Being averaged responses, several confounding factors may contribute to the shapes of the plotted curves. For this reason a multiple regression linear model was also used to quantify the independent effects of the various stimulus parameters (the Table). Several factors are evident from these plots. The mean CRFs in Figures 5A through 5D, are about log-linear and so would be well approximated by the power function

$$
R=k C^{z}
$$

where $R$ is the response, $C$ the test contrast, $k$ a scaling factor, and $z$ the exponent. The effects of the viewing distance (Figs. 5A, 5B), recording channels (Figs. 5C, 5D), and regions (Figs. $5 \mathrm{E}, 5 \mathrm{~F})$ appear to be largely multiplicative rather than additive. These factors and the heteroskadastic variance suggested that both contrast and response required log-transformation before fitting with the linear model. Both contrast and the responses were converted to decibels $\left(10 \log _{10}\right)$.

For the 9-region stimuli the mean response amplitude appears to be slightly larger at the closest viewing distance, 16 $\mathrm{cm}$, reducing as the distance increases; however, the differences are small and fall well short of scaling linearly with the 8fold change in distance (Fig. 5A). There was no apparent effect of viewing distance in the 17-region data (Fig. 5B). As for the electrode placements (Figs. 5C, 5D), both stimulus ensembles produced maximum response amplitudes on channel 3 (C-D), and minima on Channel 2 (B-Inion). The signal Fz (position A, Fig. 4) referenced to the inion (Channel 1, Fig. 4) was also large (Fig. 5C) and roughly corresponds to Fz referenced to Oz.

The area of each region of the 9-region ensemble corresponds to the sum of two regions of the 17-region stimuli 
A
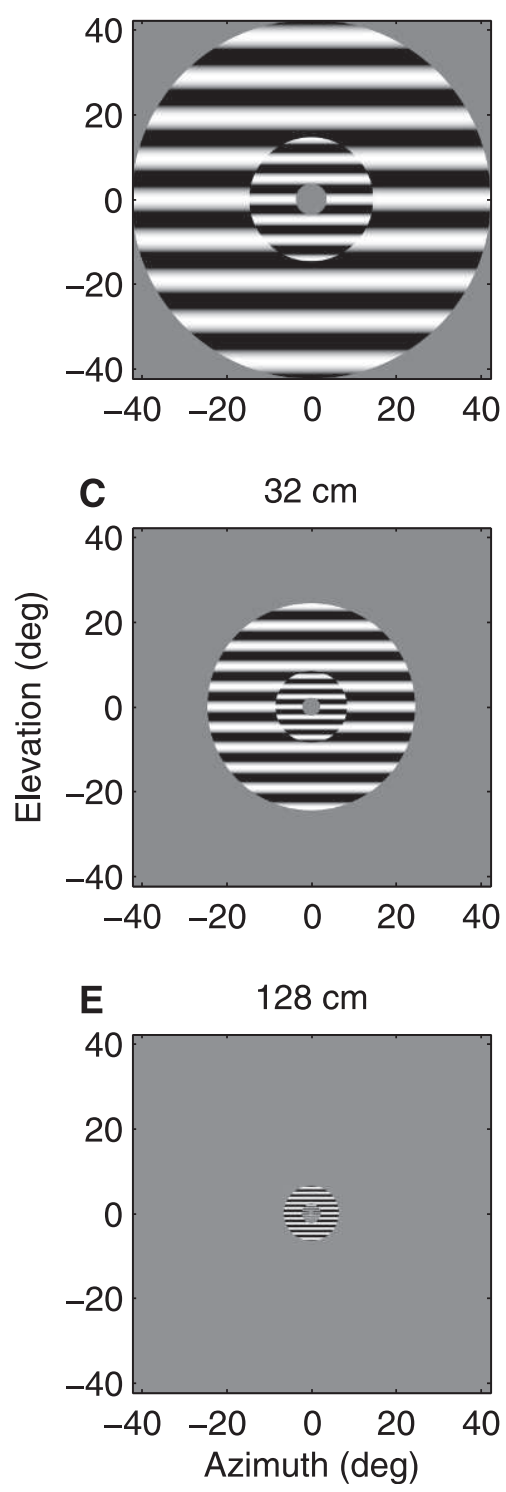

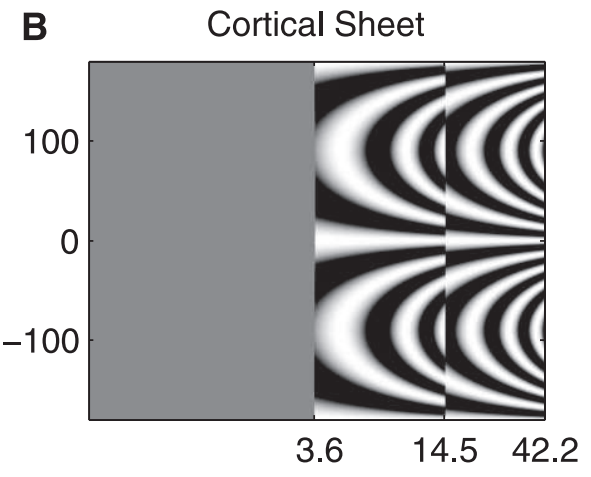

D

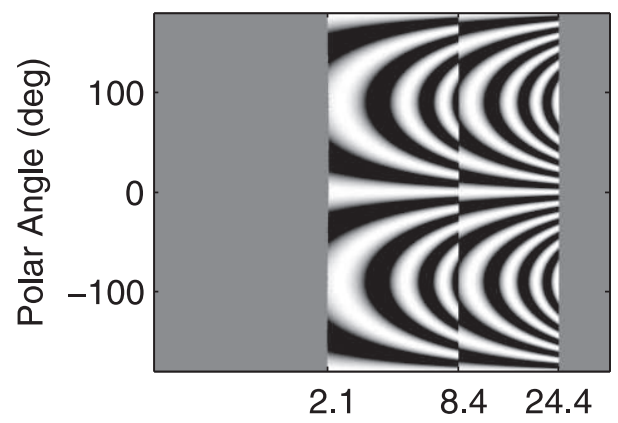

$\mathbf{F}$

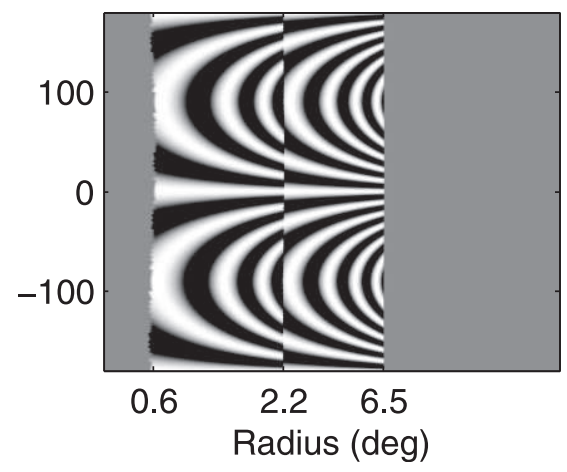

FIGURE 3. Figures illustrating the effect of increasing viewing distance on the representation in cortical area V1. On the left column are close approximations of the retinal representations of the grating stimuli used in the study (A, C, E) to stimuli as viewed at 16 , 32 , and $128 \mathrm{~cm}$. The right column (B, D, F) shows the calculated log-polar transformed versions of the stimuli at left simulating the representation on the flattened visual cortex. The transformation calculations were done with Matlab. For (B, D, F) the abscissa represents the log radius (degree) from the center of each stimulus, and the ordinate is the polar angle around each stimulus ring. For example, in (B) the abscissa labels $42.2^{\circ}, 14.5^{\circ}$, and $3.6^{\circ}$ are the radial angles subtended at the retina when viewing the outer, middle, and central ring at $16 \mathrm{~cm}$. Not only is the area of the cortical representation invariant, but the spatial frequencies of the stimuli in terms of cycles per millimeter of cortex also remains the same. Note that the negative and positive polar angles, would actually be viewed in the left and right halves of the VF, which are represented in each hemicortex.

(Fig. 1). Overall the 9-region stimuli produced larger mean response amplitudes (Fig. 5E) than the 17-region stimuli (Fig. $5 \mathrm{~F})$, but not twice as large. We, therefore, decided to incorporate the response per unit area in each part of the field in our linear model. The linear model incorporated factors for regions, sex, channel, stimulus regions (see below), and covariates for decibel contrast, viewing distance, and age. There was also a reference term, which was the grand mean across nonfitted conditions (i.e., region 1 of the 9-region protocol; and responses of males, recorded on channel 1). The factors were fitted as contrasts against the reference condition, which meant the $t$-statistics and $P$ values for the fitted items indicate the significance of the difference of the fitted values from the reference. That is the significance of female response relative to males, the other electrodes relative to electrode one, and so on. The Table shows the results of the model.

The reference value was $90.36 \mathrm{nV}$ ( $t$-statistic 58.4). There was a small but significant increase in responses with age $(0.02$ $\mathrm{dB} / \mathrm{yr}, P=0.01)$. Females had significantly larger responses than males by $2.63 \mathrm{~dB}$ ( 1.83 times), and relative to Channel 1 (reference) channels 2 and 3 had smaller responses ( 0.72 and 0.90 times), all $P$ was less than $10^{-12}$. As in previous reports, $2,3,26$ the CRFs were well represented by a power-law contrastresponse function, Response $=k \times$ Contrast $^{z}$, with an exponent less than $1,0.10 \pm 0.01$.

The Table shows that relative to region 1 only regions 8 and 9 gave significantly different responses (the Table, rows "9 Region, r2" to "r9"). In terms of comparing the 9- and 17- 


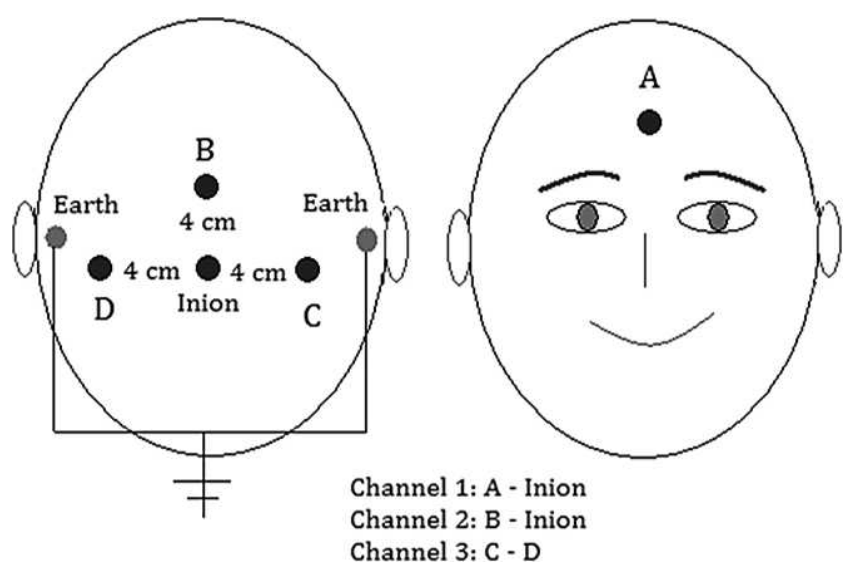

FIGURE 4. Schematic representation of the placement of electrodes that defined the configuration of the three recorded channels. The point A (front view right) illustrates an electrode, positioned on the midposition of the forehead, Fz, and $\mathbf{B}$ represents $\mathrm{Oz}$, both of the 10-20 system. Points $\mathbf{C}$ and $\mathbf{D}$ are similar to the $10-20$ points I1 and I2.

region stimuli then, as mentioned above, a reasonable thing to do would be to estimate the response per unit area. In order to accomplish this, the amplitudes of the responses to pairs of the 17-region stimuli were summed to make nine measures that sampled the equivalent parts of the $\mathrm{VF}$ as in the 9-region stimulus. These summed equivalents are termed r1s to r9s in the Table. By examining Figure 1, we can see that $\mathrm{r} 1 \mathrm{~s}$ is the sum of the responses to regions 1 and 5 of the 17- region stimuli, r2s is the sum regions 2 and 6 of the 17- region stimuli, and so on. If the responses of the summed equivalents were significantly larger than their 9-region counterparts, then the 17-region stimulus would be more efficient in generating larger responses per unit area of stimulus. The Table shows that these equivalent stimulus regions typically gave responses that were approximately 1.5 times their 9-region counterparts. Interestingly, the central stimulus of the 17-region array gave a smaller response than the same region in the 9-stimulus array $(0.54$ times), perhaps due to the large difference in stimulus frequency (Fig. 2).

The other aim of the study was to investigate the effect of changing viewing distances on the response amplitude. Viewing distance was fitted as the $\log _{2}$ values for 16,32 , and $128 \mathrm{~cm}: 4,5$, and 7 . On average responses dropped by $-0.17 \pm$ $0.03 \mathrm{~dB}$ per octave of viewing distance $\left(P<1.3 \times 10^{-7}\right)$. The fitted values correspond to $-0.17 \mathrm{~dB} ; 4,5,7=-0.68,-0.85$, $-1.19 \mathrm{~dB}$, respectively; or $1,0.96$ and 0.89 times smaller relative to the results at $16 \mathrm{~cm}$. Thus, the 8 -fold (i.e., 128/16) change in viewing distance made approximately a $10 \%$ difference in mean response amplitude (1 to 0.89 ).

The Table and Figure 5 indicated that the 17-region stimulus had small responses making a rapid test of visual function unlikely. For comparison with a companion study, which also used a 9-region MSV method, we, therefore, examined the distributions of the number of significant regions obtained for the current 9-region tests (Fig. 6). Note that the method for computing significance in the complex plane (Materials and Methods section) explicitly takes into account variability in the phase of the signals. Distributions are shown for significance at $P$ less than 0.05 and $P$ less than 0.001 .

\section{Discussion}

As reported in a previous study, ${ }^{2}$ the CRFs were roughly loglinear, being well described by a power function with an

TABLE. Fitted Values for the Reference

\begin{tabular}{|c|c|c|c|c|c|}
\hline Factor or Covariate & dB Coef & SE & $t$-Stats & $\boldsymbol{P}$ & Antilog Values \\
\hline Reference & 19.56 & 0.33 & 58.4 & $*$ & $90.36 \mathrm{nV}$ \\
\hline Age $(\mathrm{dB} / \mathrm{y})$ & 0.02 & 0.01 & 2.76 & 0.01 & \\
\hline Sex $=$ female & 2.63 & 0.09 & 29.9 & $*$ & $1.83 \times$ \\
\hline Channel 2 = B-Inion & -1.45 & 0.1 & -14.5 & $*$ & $0.72 \times$ \\
\hline Channel $3=$ C-D & -0.46 & 0.1 & -4.54 & $*$ & $0.90 \times$ \\
\hline Exponent $z$ & 0.10 & 0.01 & 10.2 & $*$ & \\
\hline 9 region, r2 & -0.34 & 0.25 & -1.36 & 0.17 & $0.92 \times$ \\
\hline 9 region, $r 3$ & -0.46 & 0.25 & -1.87 & 0.06 & $0.90 \times$ \\
\hline 9 region, $r 4$ & -0.36 & 0.25 & -1.48 & 0.14 & $0.92 \times$ \\
\hline 9 region, 15 & -0.24 & 0.25 & -0.96 & 0.34 & $0.95 \times$ \\
\hline 9 region, r6 & -0.25 & 0.25 & -1.02 & 0.31 & $0.94 \times$ \\
\hline 9 region, $r 7$ & -0.32 & 0.25 & -1.32 & 0.19 & $0.93 \times$ \\
\hline 9 region, r8 & 1.56 & 0.25 & 6.34 & $*$ & $1.43 \times$ \\
\hline 9 region, $r 9$ & 0.54 & 0.25 & 2.21 & 0.03 & $1.13 \times$ \\
\hline 17 region, r1s & 1.76 & 0.25 & 7.16 & $*$ & $1.50 \times$ \\
\hline 17 region, r2s & 1.81 & 0.25 & 7.37 & $*$ & $1.52 \times$ \\
\hline 17 region, r3s & 1.99 & 0.25 & 8.10 & $*$ & $1.58 \times$ \\
\hline 17 region, r4s & 2.06 & 0.25 & 8.40 & $*$ & $1.61 \times$ \\
\hline 17 region, r5s & 2.04 & 0.25 & 8.30 & $*$ & $1.60 \times$ \\
\hline 17 region, r6s & 1.76 & 0.25 & 7.16 & $*$ & $1.50 \times$ \\
\hline 17 region, r7s & 2.07 & 0.25 & 8.43 & $*$ & $1.61 \times$ \\
\hline 17 region, r8s & 0.13 & 0.25 & 0.51 & 0.61 & $1.03 \times$ \\
\hline 17 region, r9s & -2.71 & 0.25 & -11.03 & $*$ & $0.54 \times$ \\
\hline $\log _{2}$ (viewing distance) (db/octave) & -0.17 & 0.03 & -5.31 & * & $0.96 \times$ \\
\hline
\end{tabular}

The covariates are age, decibel contrast, $\log _{2}$ viewing distance, and factors for sex, channel, the original 9-regions (r2 to r9), and the summed responses for pairs of 17-region equivalents to the 9-region stimuli (17 region, r1s to r9s; the s indicating summed regions). The $t$ and $P$ values denote the significance of the fitted value. SE is standard error of the mean (SEM). For the covariates age and distance $\log _{2}$ the units are $\mathrm{dB} / \mathrm{yr}$ and $\mathrm{dB} /$ octave of viewing distance. The exponent $z$ is dimensionless.

* The significance $(P)$ labeled had a significance of $P$ less than $10^{-12}$. 

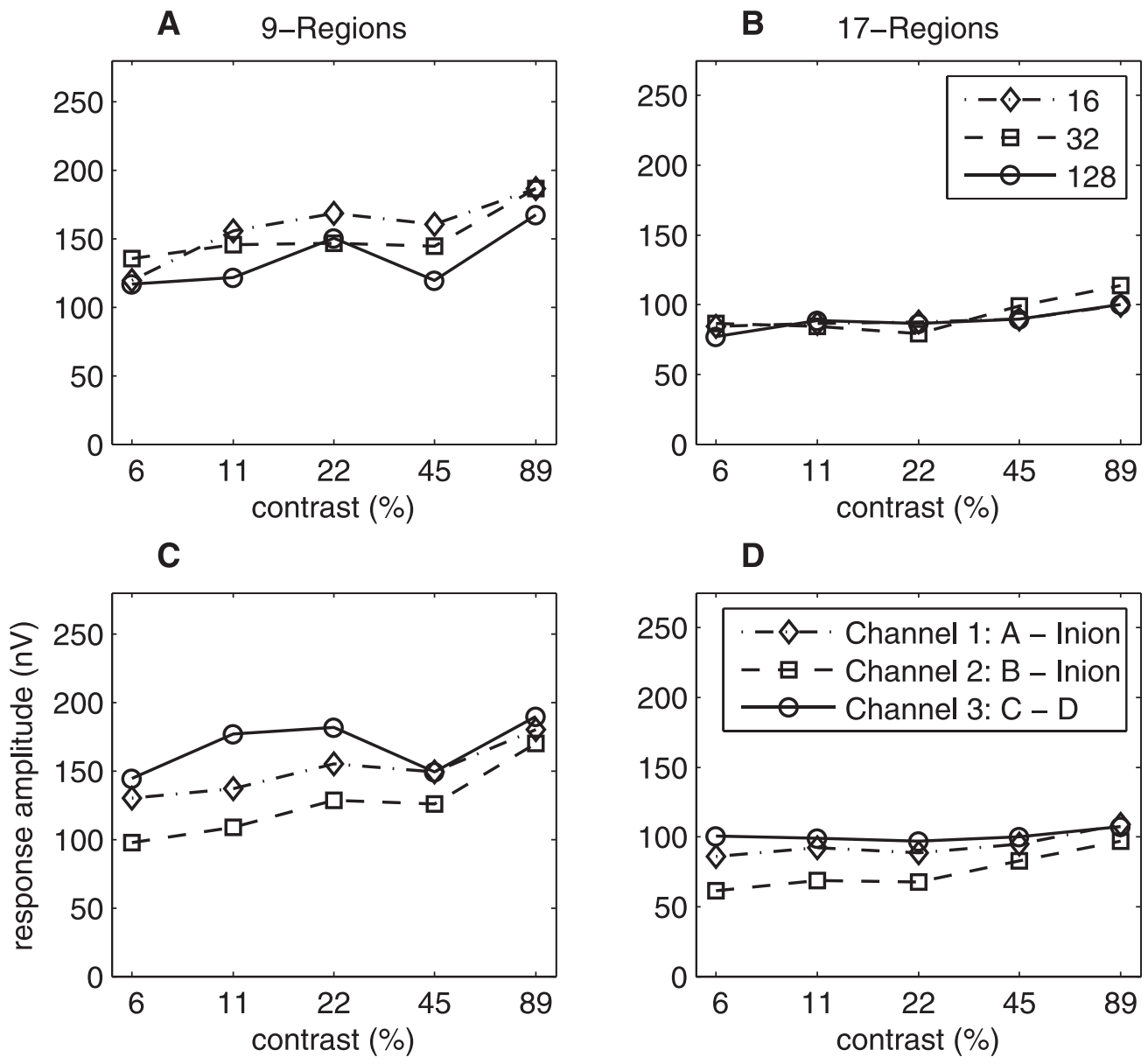

E
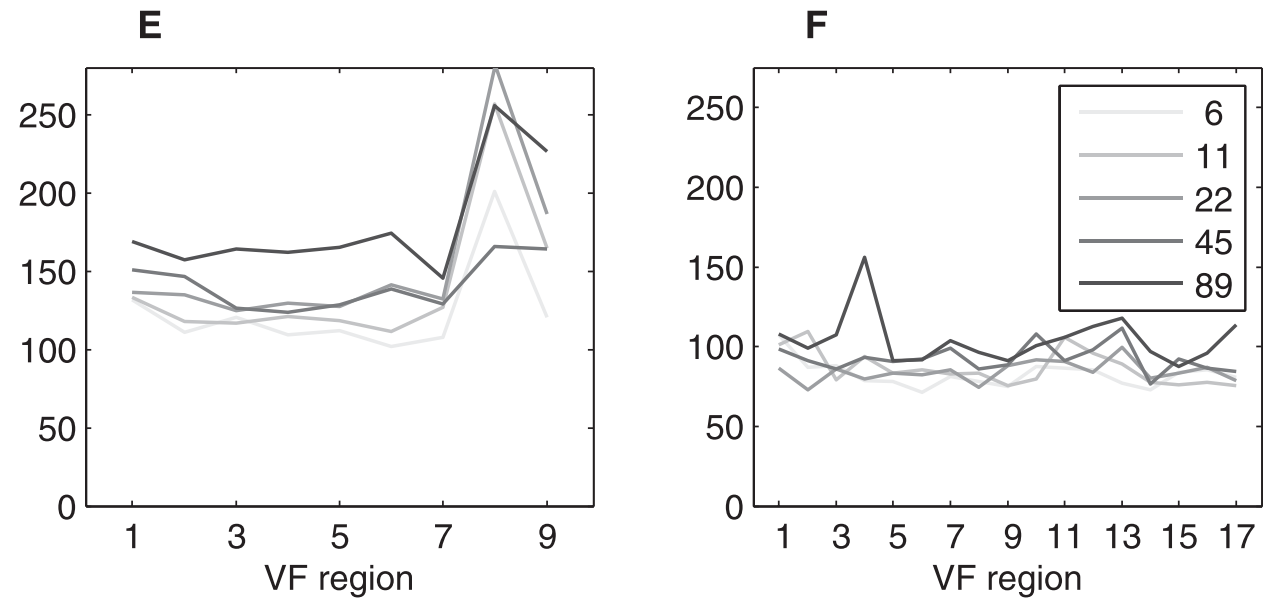

FiguRE 5. Mean responses plotted for the 9- (left column) and 17-regions (right column) stimuli. Mean response amplitude computed across. (A, B) Subjects $(n=14)$, regions, and the three electrode placements, showing the effect of viewing distance. (C, D) Means across subjects $(n=14)$, regions, and the three viewing distances, showing the mean response at the three electrodes. (E, F) Means across subjects, viewing distance, and electrode positions, resulting in one curve for each of the five contrast steps. Standard errors of the mean (SEM) bars were not plotted above to avoid clutter, however, for $(\mathbf{A}, \mathbf{C}, \mathbf{E})$, their mean values were $1.60,2.28$, and $1.62 \mathrm{nV}$; for $(\mathbf{B}, \mathbf{D}, \mathbf{F})$, these were $0.62,1.30$, and $0.87 \mathrm{nV}$.

exponent around 0.1 , that is straight lines with shallow slopes when plotted on a log-contrast abscissa (Figs. 5A-5D). These CRFs are also similar to those reported in other mfVEP studies using either pseudorandom pattern-reversal stimuli, ${ }^{26,27}$ pseudorandom flash stimuli, ${ }^{28}$ or conventional steady-state VEPs. ${ }^{29}$ This behavior may originate from the saturating responses of M-cells. ${ }^{30}$ Our result of larger responses for females concurs with findings from other pattern mfVEP studies, ${ }^{31}$ which is thought to be related mainly to skull thickness. ${ }^{32}$
As expected for responses arising from a log-polar representation of the visual world (Fig. 3), viewing distance had little effect on response size, changing by just $10 \%$ over an 8 -fold change in viewing distance, $-0.17 \pm 0.03 \mathrm{~dB}$ per octave (the Table). The results are similar to those reported by Hood et al. ${ }^{33}$ who used three concentric rings of 18-multifocal stimuli with similar angular subtenses and viewing distances to those used here. Results were presented for three subjects, but provided no explanation of the lack of response change with 


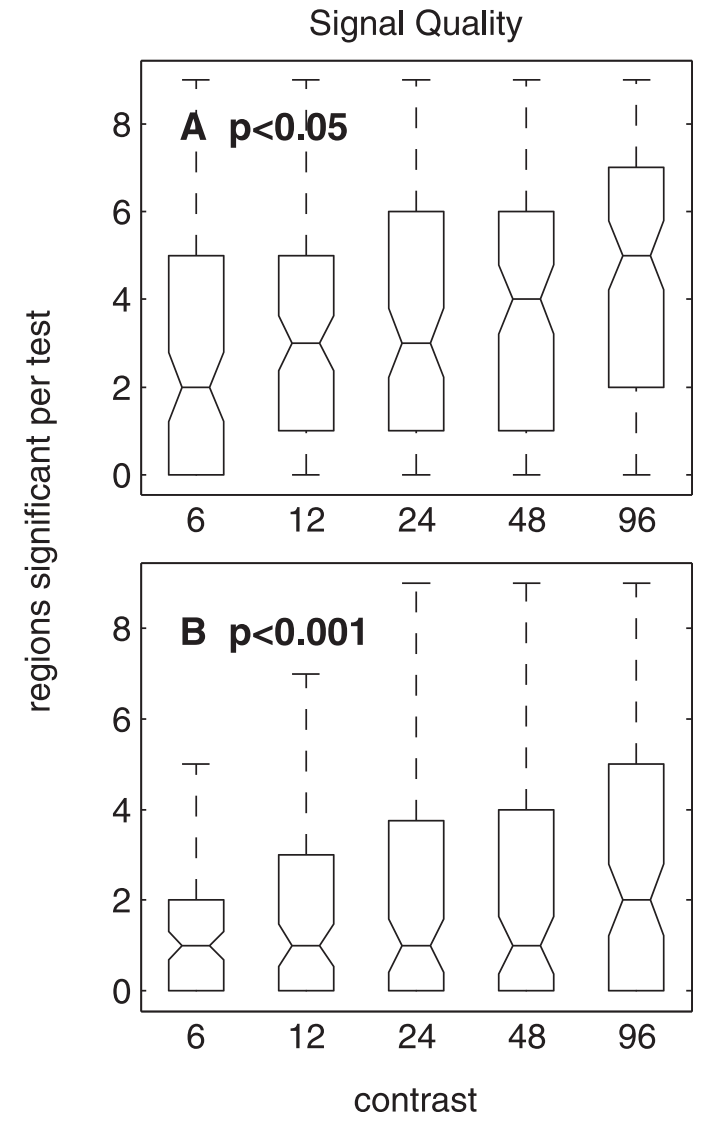

FiguRE 6. Box plots indicating the number of tests that contained significant responses for two repeats of the 20 second 9-region stimuli. The value at the notch is the median, the upper and lower box edges are the 25th and 75th percentiles, and the whiskers mark the 5th and 95th percentiles. (A) At the $P$ less than 0.05 level there was little difference in the distribution of significant responses between contrasts 0.24 and 0.96 . (B) A surprising number of responses were significant even at $P$ less than 0.01 . Significance levels were based on Fstatistics that took into account consistence of response phase (Materials and Methods section).

viewing distance. The small dependence on viewing distance observed in both studies might be due to a small departure from a strict log-polar representation, especially nearer the fovea. ${ }^{16-19}$ The results may be clinically useful in the sense that they reveal that multifocal stimulus ensembles could be zoomed in size to explore different parts of the VF without a serious degradation of response size.

The difference in magnocellular $(\mathrm{M})$ and parvocellular $(\mathrm{P})$ cell densities as a function of eccentricity might have been expected to change the contrast-response function shape, at least for the farthest viewing distance. Baseler and Sutter, ${ }^{1}$ provided data suggesting that the largest VEP differences produced by differences in $\mathrm{M} / \mathrm{P}$ density occur for eccentricities less than $1.5^{\circ}$ and contrasts below $15 \%$. A possibly related effect is a small dip in the contrast-response function of MSV stimuli around $7 \%$ contrast that was largest for the central stimulus. ${ }^{2}$ This might restrict the use of scaling of MSV arrays to those that are mostly larger than $5^{\circ}$ and $15 \%$ contrast. It is worth mentioning that the contrast responses functions for Baseler and Sutter's M-component was very flat, like those reported here.

In terms of response per unit area, the pairs of 17-region stimuli produced responses that were approximately $50 \%$ larger than the 9-region stimuli of the same area. In fact, one might have expected the responses of the pairs of 17-region stimuli to be less than their 9-region counterparts due to the different temporal frequencies used for each pair. This raises the issue of how large the frequency effects were. In two previous studies, ${ }^{2,3}$ we showed small but significant frequency dependent change in the sort of range used here, of approximately $1 \mathrm{uV} / \mathrm{Hz}$. For the two stimulus arrays used here the responses for different regions were quite similar at all contrasts (Figs. 5E, 5F), indicating the scaling of the stimuli was reasonable. Notable exceptions were regions 9 and 8 of the 9region array, the central region, and one peripheral region (Fig. 1). Such fluctuations are evident even when the experimental design allows separate frequency and regional effects to be estimated, ${ }^{3}$ but seem to be reduced when higher spatial frequencies are used in the stimulus regions. ${ }^{4,5}$ Much of the remaining variation in Figures $5 \mathrm{E}$ and $5 \mathrm{~F}$ are due to overall, smaller responses in the superior field, although most are not significant (the Table). ${ }^{3}$

In any case, it would appear that the frequency effects are small and so we interpret the improvement in the response/ stimulus area as being mainly due to cortical folding effects. Interestingly, the present results mirror a companion study, in which the summed responses of the same 9-regions stimulus used here were larger than the response to a single large stimulus that had the same area as the 9-region ensemble. ${ }^{34}$

That companion study ${ }^{2}$ indicated that two repeats of the 20 second 9-region stimulus was adequate to estimate models like those used here, but that for VF testing perhaps 8 to 10 repeats would be needed. The study examined the number of significant responses and also the sum of those responses to look at signal quality. Here, we examined the distribution significant results (Fig. 6) and have come to similar conclusions. The 17-region test, with its smaller responses, would perhaps take too long for a clinically useful test. Across the two repeats responses were averaged in the complex plane. We, therefore, used F-static based significance testing ${ }^{4,5}$ to take into account that the real and imaginary axes represent two independent random variables. Thus, the significance testing took into account phase variability, random signals having random phase do not add constructively across averages.

The method has shown very consistent phase repeatability in our other studies. ${ }^{2,3}$ One of those studies was very similar to the one presented here, and contained a great number of repeats allowing phase stability to be quantified. ${ }^{3}$ The stimuli were at $95 \%$ contrast and had no central region, but the spatial frequencies and layout of the remaining eight regions were like those in this study for a viewing distance of $25.6 \mathrm{~cm}$. Three monocular conditions were repeated at stimulus frequencies in the range 15.45 to $16.67 \mathrm{~Hz}$, and three in the range 16.84 to $21.51 \mathrm{~Hz}$. Four repeats were done on each of those six tests, and that was repeated on 16 subjects. The whole process was repeated again on a second visit, and the recording electronics were identical to the present study. To examine phase stability here the SD for the four repeats per test was calculated. Figure 7 shows box plots for resulting 384 SDs $(16 \times 3 \times 8)$ for each visit and frequency band. The median SD was approximately $12^{\circ}(1 /$ 30 of a cycle), and the 90th percentiles were in the range $38.5^{\circ}$ to $51.2^{\circ}$ (1/9.4 to $1 / 7.0$ cycle)

We have not described the outcomes for frequencies that were designed to capture nonlinear interactions between the stimulus regions. Two earlier MSV studies ${ }^{2,3}$ have shown that these are very small and better recorded by electrodes placed to be biased toward extrastriate cortical responses. The important message for this study is that these small interactions did not overlap with the main second harmonics responses.

The stimuli used in this study resemble those of the FDT perimeter, in producing the spatial FD illusion, ${ }^{6}$ and so might have some utility in measuring the effects of glaucoma. 


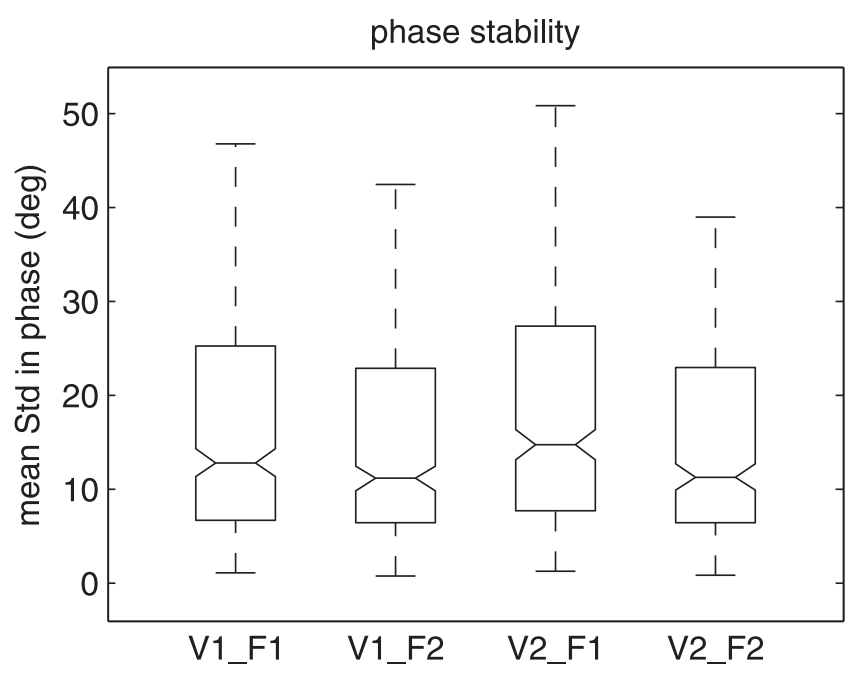

Figure 7. Box plots of the SDs in the phase of each of eight frequencies per test in 192 MSV tests, which had four repeats per recording session. A set of three tests was repeated on 16 subjects, two visits, and at two frequency bands on each visit ( $\mathrm{F} 1=15.45$ to 16.67 $\mathrm{Hz}, \mathrm{F} 2=16.84$ to $21.51 \mathrm{~Hz}$ ). Each of the four box plots is, therefore, based on 384 SDs (1536 total). All the tests had contrast $95 \%$ and the spatial frequencies were as if the present monitor of the present study was viewed from a distance of $25.6 \mathrm{~cm}$. The higher temporal frequency bands showed somewhat lower variance on each visit.

Certainly the ERG version showed some promise in that area. 4,5 The FD illusion occurs when stimuli are presented at spatial frequencies of less than $1 \mathrm{cpd}$ that are rapidly contrast modulated at greater than $10 \mathrm{~Hz}$. The effect was initially attributed to a nonlinear $\mathrm{M}_{\mathrm{y}}$ subgroup of the M-system, ${ }^{21,35}$ but more recently, others have attributed it to other nonlinearities. ${ }^{36-39}$ That being said, an anatomic substrate for primate $\mathrm{M}_{\mathrm{y}}$-cells has recently been reported. ${ }^{40,41}$ In any case, the stimuli appear to preferentially stimulate large ganglion cells, which by virtue of their low coverage factor, may mean cell death is more easily measured. ${ }^{35}$

\section{Conclusions}

Responses to low contrasts gave responses approximately as large and reliable as responses to high contrasts. When assessing glaucomatous VF damage contrasts that result in saturation could mask some VF defects, so using less saturating contrasts might be prudent. Although the response per unit area was larger for the summed 17-region stimulus ensemble, the absolute response sizes were smaller than the 9-region ensemble. As expected, varying the viewing distance did not greatly affect the amplitude of the recorded responses. This should allow peripheral and (relatively) central disease to be tested with mfVEPs that exploited zoomed versions of the stimulus array.

\section{References}

1. Baseler HA, Sutter EE. M and P components of the VEP and their VF contribution. Vis Res. 1997;37:675-690.

2. Abdullah SN, Vaegan, Boon MY, Maddess T. Contrast-response functions of the multifocal steady-state VEP (MSV) [published online ahead of print March 21, 2012]. Clin Neurophysiol. doi: 10.1016/j.clinph.2012.02.067.

3. Rosli Y, Maddess T, Dawel A, James AC. Multifocal frequencydoubling pattern visual evoked responses to dichoptic stimulation. Clin Neurophysiol. 2009;120:2100-2108.
4. Maddess T, James AC, Goldberg I, Wine S, Dobinson J. Comparing a parallel PERG, automated perimetry, and frequency doubling thresholds. Invest Ophthalmol Vis Sci. 2000; $41: 3827-3832$.

5. Maddess T, James AC, Goldberg I, Wine S, Dobinson J. A spatial frequency doubling illusion-based pattern electroretinogram for glaucoma. Invest Ophthalmol Vis Sci. 2000;41:3818-3826.

6. Kelly DH. Frequency doubling in visual responses. $J$ Opt Soc Amer. 1966;56:1628-1632.

7. Jeffreys D. Cortical source locations of pattern-related visual evoked potentials recorded from the human scalp. Nature. 1971;229:502-504.

8. Di Russo F, Martinez A, Sereno MI, Pitzalis S, Hillyard SA. Cortical sources of the early components of the visual evoked potential. Hum Brain Mapp. 2001;15:95-111.

9. Di Russo F, Pitzalis S, Spitoni G, et al. Identification of the neural sources of the pattern-reversal VEP. Neuroimage. 2005; 24:874-886.

10. James A. The pattern pulse multifocal visual evoked potential. Invest Ophthalmol Vis Sci. 2003;44:879-890.

11. Carney T, Ales J, Klein SA. Combining MRI and VEP imaging to isolate the temporal response of visual cortical areas. SPIE. 2008;6806:680808-1-680808-28.

12. Daniel PM, Whitteridge D. The representation of the visual field on the cerebral cortex in monkeys. J Physiol. 1961;159: 203-221.

13. Hubel DH, Wiesel TN. Receptive fields, binocular interaction and functional architecture in the cat's visual cortex. J Physiol. 1962;160:106-154.

14. Hubel DH, Wiesel TN. Spatial and chromatic interactions in the lateral geniculate body of the rhesus monkey. $J$ Neurophysiol. 1966;29:1115-1156.

15. Wassle H, Grunert U, Rohrenbeck J, Boycott BB. Cortical magnification factor and the ganglion-cell density of the primate retina. Nature. 1989;341:643-646.

16. Tootell RB, Switkes E, Silverman MS, Hamilton SL. Functional anatomy of macaque striate cortex. II. Retinotopic organization. J Neurosci. 1988;8:1531-1568.

17. Azzopardi P, Cowey A. The overrepresentation of the fovea and adjacent retina in the striate cortex and dorsal lateral geniculate nucleus of the macaque monkey. Neuroscience. 1996;72:627-639.

18. Schwartz EL. Computational anatomy and functional architecture of striate cortex: a spatial mapping approach to perceptual coding. Vision Res. 1980;20:645-669.

19. Adams DL, Horton JC. A precise retinoscopic map of primate striate cortex generated from the representation of angioscotomas. J Neurosci. 2003;23:3771-3789.

20. Zemon V, Ratliff F. Visual evoked potentials: evidence for lateral interactions. Proc Natl Acad Sci U S A. 1982;79:57235726.

21. Maddess T, Hemmi JM, James AC. Evidence for spatial aliasing effects in the Y-like cells of the magnocellular visual pathway. Vision Res. 1998;38:1843-1859.

22. Rosli Y, Maddess T, Bedford SM. Low spatial frequency channels and the spatial frequency doubling illusion. Invest Ophthalmol Vis Sci. 2009;50:1956-1963.

23. Hood DC, Zhang X, Hong JE, Chen CS. Quantifying the benefits of additional channels of multifocal VEP recording. Doc Ophthalmol. 2002;104:303-320.

24. Klistorner A, Graham SL. Objective perimetry in glaucoma. Ophthalmology. 2000;107:2283-2299.

25. Hoffmann MB, Bach M. The distinction between eye and object motion is reflected by the motion-onset visual evoked potential. Exp Brain Res. 2002;144:141-151.

26. Maddess T, James AC, Bowman EA. Contrast response of temporally sparse dichoptic multifocal visual evoked potentials. Vis Neurosci. 2005;22:153-162. 
27. Baseler HA, Sutter EE. M and P components of the VEP and their visual field contribution. Vis Res. 1997;37:675-690.

28. Klistorner A, Crewther DP, Crewther SG. Separate magnocellular and parvocellular contributions from temporal analysis of the multifocal VEP. Vision Res. 1997;37:2161-2169.

29. Bagolini B, Porciatti V, Falsini B. Binocular interaction and steady-state visual evoked potentials. I. A study in normal subjects and in subjects with defective binocular vision. Graefes Arch Clin Exp Ophthalmol. 1988;226:401.

30. Kaplan E. The M, P, and $\mathrm{K}$ pathways of the primate visual system. In: Chalupa LM, Werner JS, ed. The Visual Neurosciences. Cambridge, MA: MIT Press; 2004:481-493.

31. Klistorner AI, Graham SL. Electroencephalogram-based scaling of multifocal visual evoked potentials: effect on intersubject amplitude variability. Invest Ophthalmol Vis Sci. 2001;42: 2145-2152.

32. Mitchell KW, Howe JW, Spencer SR. Visual evoked-potentials in the older population-age and gender effects. Clin Phys Physiol Meas. 1987;8:317-324.

33. Hood DC, Ghadiali Q, Zhang JC, Graham NV, Wolfson SS, Zhang X. Contrast-response functions for multifocal visual evoked potentials: a test of a model relating V1 activity to multifocal visual evoked potentials activity. J Vis. 2006;6:580593.
34. Vaegan, Rahman AMA, Sanderson GF. Glaucoma affects steady state VEP contrast thresholds before psychophysics. Optom Vis Sci. 2008;85:547-558.

35. Maddess T, Henry GH. Performance of nonlinear visual units in ocular hypertension and glaucoma. Clin Vis Sci. 1992;7:371383.

36. White AJR, Sun H, Swanson WH, Lee BB. An examination of physiological mechanisms underlying the frequency-doubling illusion. Invest Ophthalmol Visual Sci. 2002;43:3590-3599.

37. Anderson AJ, Johnson CA. Mechanism isolated by frequencydoubling technology perimetry. Invest Ophthalmol Vis Sci. 2002;43:398-401.

38. Morgan JE. Selective cell death in glaucoma: does it really occur? Br J Ophthalmol. 1994;78:875-879. discussion 879880.

39. Dacey DM. Morphology of a small-field bistratified ganglion cell type in the macaque and human retina. Vis Neurosci. 1993;10:1081-1098.

40. Crook JD, Peterson BB, Packer OS, et al. The smooth monostratified ganglion cell: evidence for spatial diversity in the Y-cell pathway to the lateral geniculate nucleus and superior colliculus in the macaque monkey. J Neurosci. 2008; 28:12654-12671

41. Petrusca D, Grivich MI, Sher A, et al. Identification and characterization of a Y-like primate retinal ganglion cell type. $J$ Neurosci. 2007;27:11019-11027. 